Journal of Computer Science 6 (12): 1511-1517, 2010

ISSN 1549-3636

(C) 2010 Science Publications

\title{
A Simulative Study on the Performance Evaluation for Simultaneous and Successive Mobility for Mobile IPv6
}

\author{
Ibrahim Abdullah and Mohamed Othman \\ Department of Communication Technology and Networks, \\ University Putra Malaysia, 43400 UPM Serdang, Selangor, Malaysia
}

\begin{abstract}
Problem statement: Mobile Internet Protocol (MIP) is the forms of the backbone for next generation wireless internet technology to provide uninterrupted network service while a node on the move. The original MIP use Home Agent to forward the traffic. Thus, it does not have problem with simultaneous mobility, i.e., the special case when both end nodes are mobile and move at about the same time. However, MIPv6 uses location binding updates which sent directly to a correspondent node to complete its registration. Thus, MIPv6 is vulnerable to the simultaneous mobility problem which leads to the interruption of communication and cause messages lost, especially protocol messages. Approach: This article analyzed the simultaneous and successive fast mobility problems and handover latency. Then propose a mechanism by adopting technique to the mobile node's Home Agent. Results: We simulated the proposed scheme using ns-2 and we present and analyze the performance testing for the proposed scheme by comparing it with the conventional MIPv6. Conclusion: Simulation results showed this scheme can effectively resolve simultaneous and successive mobility problems and reduce the handover latency which is affected by long registration time.
\end{abstract}

Key words: MIPv6, simultaneous mobility, successive mobility, return routability, handover, DAD

\section{INTRODUCTION}

Mobile IP is the dominant mobility handling protocol for host mobility on the Internet. However, with the rise of IPv6, a new mobility handling protocol was needed for host mobility on IPv6 networks. The new protocol, proposed by the Internet Engineering Task Force is Mobile IPv6 (IETF-mobileip, 2004). Among the new features introduced with MIPv6 is a Route optimization which allowed a direct communication between end nodes, but a major problem is that it makes MIPv6 vulnerable to the simultaneous mobility problem; which is the case when both end nodes are mobile and they move at about the same time. Therefore, the mobility signaling for location registration are lost during simultaneous mobility, this problem may arise because a particular mobility protocol was designed with the implicit assumption that the correspondent node $(\mathrm{CN})$ does not move when the mobile node (MN) moves. Simultaneous mobility problem becomes a critical issue currently, when the two mobile nodes move at about the same time both use route optimization procedure to exchange the mobility messages. In the location registration through binding update phase, the route optimization-related signaling (Johnson et al., 2004), such as Home Test Init message (HoTI) and Care of Test Init message (CoTI) are sent to the old IP address, this results route optimization messages lost. It may cause communications disruption between the MNs or even cannot be resumed, which will affect the normal function of the network. In addition, to solve simultaneous mobility issue the successive fast mobility must be taken into consideration. This case, when a MN moves to a new network and during its location registration to its home agent (HA) and to its $\mathrm{CN}$, however, before these registrations complete, the MN moves again to a new network. At the same time, we must consider the handover performance improvement and packet loss reduction enhancements to satisfy the requirement of the real-time and non-real time application services. This article proposed adopting technique scheme to the MN's home agent to solve simultaneous mobility problem and improve the MN handover performance, as well as reduce the packet loss. This scheme relies on the assumption that a MNs always moves in several range limited networks.

The next discussion presents a detailed analysis of related study. Subsequently, an overview of simultaneous mobility problem followed by

Corresponding Author: Ibrahim Abdullah, Department of Communication Technology and Networks, University Putra Malaysia, 43400 UPM Serdang, Selangor, Malaysia 
descriptions of the handover latency and proposed scheme in detail. Then present performance metrics used to evaluate the proposed scheme and present the simulation model and result prior to the study being concluded.

Related study: performance analysis is an important part of research for proving different theories and models. In this article we will present an analytical and simulation studies which cover two aspects; simultaneous mobility as well as successive fast mobility and handover performance.

There are few solutions to deal with first aspect; simultaneous mobility problems in the IP layer like using two kinds of fixed proxies to keep the messages and proxies for time $T_{h}$ and then forward that messages, otherwise, using Corresponding Node (CN)'s Home Agent (HA) to forward that messages. Therefore, after receiving these three control messages the CN's HA keeps the messages for a short time $\mathrm{T}_{b}$ while forwards them as in (Wong et al., 2003a).

The analytical research study of signaling to home address has been introduced by Wong and Duttay (2005), which is more MIPv6-centric and does not involve query/response messages between HAs. Unlike the solution with stationary proxies, MN-A's HA does not have to be involved. The MN-A just sends control messages directly to MN-B's home address not the Care-of-Address (CoA). Thus, MN-B's HA will take care of forwarding the binding update to MN-B. However, this smart re-forwarding requiring modification to MN's home agent as well as the mobile node and also it has increase in latency in the control signaling also arise anew issue for the small interval time when HA does not yet have the latest care-of address of MN.

Analyze previous solutions has been consider to prove some new theorems and propose new solution. Analytical framework used in Wong et al. (2007) show in the first solution; Forwarding proxy in previous network for MIPv6 ordinary routers, in the previous network would need to be extended with forwarding proxy functionality. This would involve significant modifications to MIPv6. However, getting common routers to do this kind of forwarding might be considered an unacceptable demand.

Combination of sender-side and receiver-side mechanisms consider as a second solution; a combination of sender-side proactive binding update proxies and receiver-side pro-active redirecting location proxies was proposed as a general solution in (Wong et al., 2003b) that was applied as a solution to SIPMM and MIP-LR. Here they propose that it can also be applied to MIPv6.
The HAs of the sender and receiver, can serve the proactive binding update proxy and pro-active redirecting location proxy. Return rout ability procedure has to be modified so the CoTI message goes through the sender's HA. Another modification to MIPv6 is that the binding update must first be reversed tunneled to a MN's own HA before being forwarded to the correspondent node. The selection of period to keep a copy of such message and for a query/response should be carefully chosen based on reasonable estimates of the appropriate signaling and computational delays of the network. The proposed receiver-side mechanisms only, allow MN-A to sends all control messages to MN$B$ using MN-B's home address, thus forcing MN-B's HA to be involved. MN-B's HA will act as a pro-active forwarding location proxy, forwarding the control message to MN-B as usual, but keeping a copy of it for time. If it gets any binding updates from MN-B during that time, it pro-actively forwards the message to $\mathrm{MN}$ B. This proposed method adds forwarding proxy in the previous network with substantial functionality is not desirable and is not good in that they require significant changes to MIPv6.

Wong et al. (2003a) introduced a framework for handling simultaneous problems. By suggest two ways the first one; forwarding mechanisms from previous network since the lost packets in simultaneous mobility situations are going to the previous network (associated with the old $\mathrm{CoA}$ ), the forwarding mechanism would intercept packets destined to the old CoA and forward them to the new CoA.

There are no foreign agents in MIPv6, so it is inapplicable to add a forwarding mechanism from the previous network, either a new entity is needed, or the local router needs to take on this functionality. The second by using stationary proxies that used two kinds of stationary proxies that is responsible for obtaining the latest CN's location for MNs and maintaining the latest MN's location and delivering such information to the MN's CN. But, in order to use stationary proxies with MIPv6, additional modifications are needed to MIPv6 also the query/response functionality that would be significant addition to HA functionality.

When successive mobility occurs in the network, the two solutions about simultaneous mobility problems above cannot study normally. In those schemes, if $\mathrm{CN}$ moves before $\mathrm{MN}$ finishes correspondent registration and home registration doesn't finish as well, simultaneous problem still exists. These researches only focus on the performance of handover, which have not resolve the simultaneous mobility and it modified the operation in both link layer and network layer mostly, which is not fixed the original aim of MIPv6. 


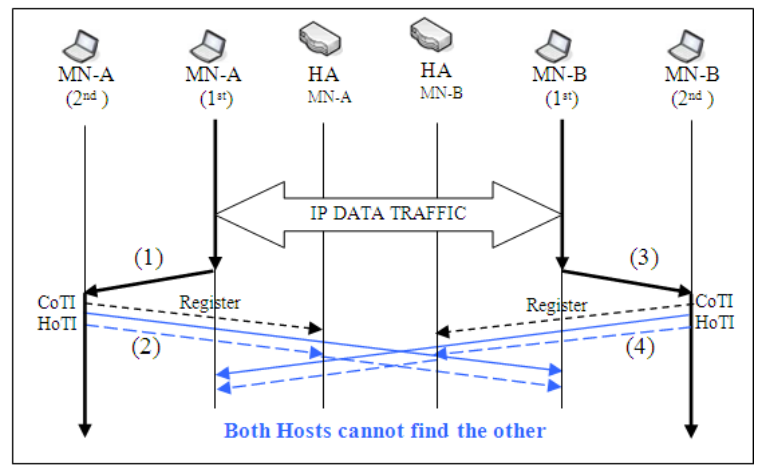

Fig. 1: Both MNs does not complete RRP process

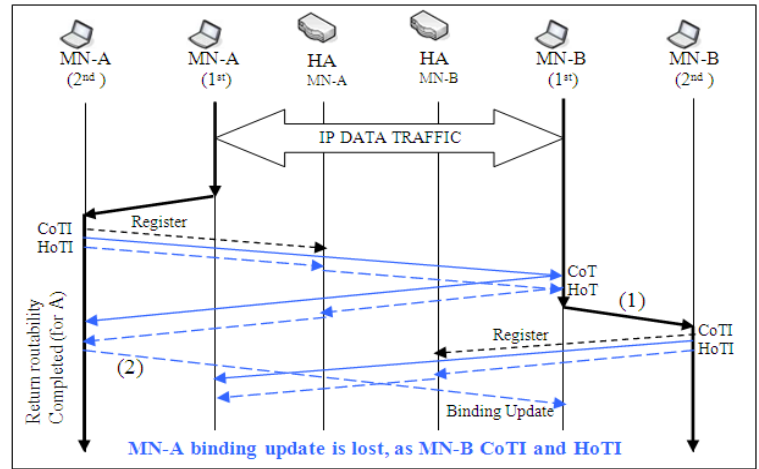

Fig. 2: Only one MN completes the RRP process

There are several approaches proposed to deal with second aspect; handover latency and packet loss in wireless overlay networks (Soliman et al., 2005; Badis and Agha, 2003; Ma and Fang, 2004). Avoiding packet losses using buffering and simultaneous binging to adjacent routers are also proposed and analyzed in (Salamah et al., 2003). In addition, some researchers are interested in assignment strategies for choosing between various communication layer and network architectures (Klein and Han, 2004).

Simultaneous mobility overview: When a MN and its $\mathrm{CN}$ is mobile node and they are move at the same time, messages will be lost in the Return Routability Procedure (RRP) or later. Those would lead to the failure of correspondent registration. When an MN moves to a new foreign network, during Initiation of registration with the HA, any movement of the other node does not matter. However, when it comes to the correspondent registration the situation is changes, because it is depends for its success upon messages reaching the other node. If the other node moves any time before receiving the binding update, one or more messages may be lost and the correspondent registration procedure may therefore fail. The messages will lost may be during the return routability procedure phase or after. The two cases (as describe in Wong et al., 2007), as follows:

Case I: When message lost during Return Routability Procedure (RRP), as shown in the Fig. 1, the two MNs are moving at the same time and both can't finish correspondent registration, result on binding procedure fails, as in following step:

- When the MN-A moved it will initiate the RRP with a MN-B

- MN-A sends HoTI and CoTI messages to MN-B. In this case, HoTI or CoTI may be lost because they are sent to the MN-B's old CoA

- On the other side, MN-B initiates the RR procedure with MN-A after moving

- MN-B sends HoTI and CoTI messages to the MNA's old CoA, the messages will lost

Case II: When message lost after return routability procedure, as shown in the Fig. 2 the following steps describe this case:

- MN-B moved after MN-A's return routability procedure has completed

- Then the MN-A will send binding update message to MN-B to update it is location. However, resulting to MN-B's movement, MN-A's binding update message goes to MN-B's old CoA and lost

So, MN-A's correspondent registration with binding update can't complete and MN-B will also be unable to complete its return routability procedure, resulting on binding procedure fails as shown in Fig. 2.

Handover latency overview: There are three main components of handover procedure which caused latency in MIPv6 (Malki et al., 2002; Narten et al., 1998). These components are:

- Link layer establishment delay: this the time need for the MN's physical interface to change its single connection from old access router to the new access router

- The rendezvous time delay: refer to the time need for a $\mathrm{MN}$ to hear beacons from a new access router after moving out of the old access router coverage

- Registration delay: which start when the MN sends a binding update to the new access router and finishes when the first packet arrives via the new router (Daley et al., 2003) 
Table 1: Standard handover latency for Mobile IPv6

\begin{tabular}{lllcl}
\hline $\begin{array}{l}\text { Link layer } \\
\text { establishment } \\
\text { time (sec) }\end{array}$ & $\begin{array}{l}\text { Rendezvous } \\
\text { time (sec) }\end{array}$ & \multicolumn{3}{l}{ Registration time (sec) } \\
\hline L2 handover & $\begin{array}{l}\text { Router } \\
\text { discovery }\end{array}$ & DAD & Send & Receive \\
0.05 & 0.10 & 1.00 & 0.07 & BAck \\
& & & & 0.07 \\
\hline
\end{tabular}

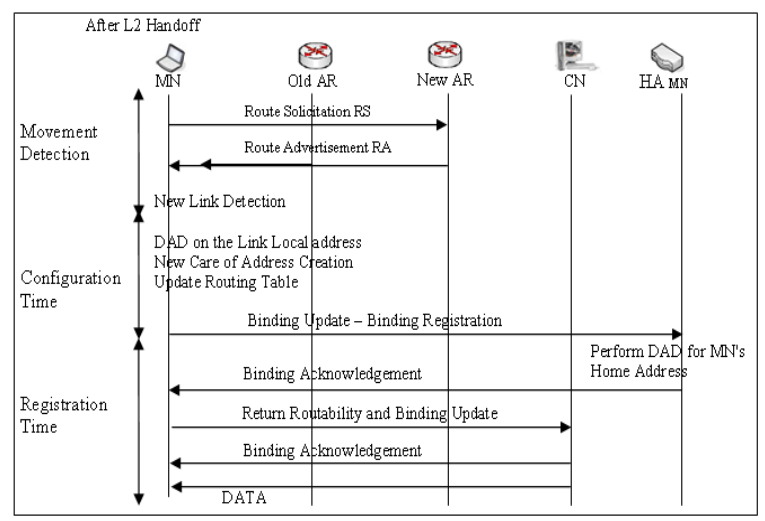

Fig. 3: Mobile IPv6 handover signaling flow

Table 1 show the typical values of each handover component in MIPv6 refer to (Lai and Chiu, 2005). It shows that registration time is a major part in the total handover latency. The basic steps involved with MIPv6 network layer handover, when a MN travels from the current access router (AR) to the new AR using generic movement detection as shown in the Fig. 3, as follows:

- $\quad \mathrm{MN}$ sends Router Solicitations (RS) to new AR

- MN receives new Router Advertisements (RA) from new AR

- MN detects that it is on a new link and performs Duplicate Address Detection to ensure that none of its interfaces is duplicated on link local address

- MN performs new CoA configuration

- $\quad \mathrm{MN}$ sends Binding Update (BU) to HA

- HA performs DAD for MN's Home Address and sends Binding Acknowledge to MN

- MN completes Return Rouetability Procedure (RRP) and sends BU-CN for Route Optimization

- $\mathrm{CN}$ returns a Binding Acknowledgement to MN and $\mathrm{MN}$ starts to receive packets

\section{MATERIALS AND METHODS}

In this article we analyze the simultaneous and successive mobility problems and the affect of the handover latency. Furthermore, propose a method which modifies some signaling flow for the conventional MIPv6 protocol and perform these signaling messages in advance prior $\mathrm{MNs}$ actual movement and it can resolve such problem and improve the overall handover performance.

Proposed scheme: In the MIPv6 the main reason of loss message is that the Home Agent (HA) has not forward the packet to the new CoA. In addition the registration time represents a large part of handover latency. The proposed method adopts technique to the MN's home agent to resolve simultaneous and successive fast mobility problems on a fixed mobile network. Therefore, under this fixed administrative management the MN's CoA can be getting previously before the actual movement of an MNs depending on receiving Link Going Down (LGD) trigger event which is based on the signal strength power below a certain predefined threshold, then MN's home agent will forward the control packet to all the available neighbor access router's CoAs in advance prior MN movements and during the process of checking the uniqueness of this $\mathrm{CoA}$ on the new subnet (i.e., by duplicate address detection mechanism) the MNs can receive all control packet, thus, reducing MN's registration time.

In this scheme, the MN in advance inform its Home Agent (HA) about its expected movement to new subnet, in order to acquire all neighbors access router's CoA; by add $a$ flag $b$ bit in the reserved field of binding update message (Liu et al., 2006) and set its value to one (i.e., $\mathrm{BU}$ messages are used for ordinary purposes if the B bit is reset). The neighbor's CoAs which need to bound are put in the home agent's binding cache list. This indicates home agent may have all CoAs of the neighbor's access router in its network. All CoA's lifetime in the home agent cache list assumed to have fair and enough time value to make an efficient reuse the same CoA, which save the time to get new $\mathrm{CoA}$ in case if the MN moves back to previous visited subnet. The MN's binding list must maintain all valid CoAs depending on the assumed lifetime. Thereafter, the HA when receiving a routability protocol message it should make multiple copies of this message and forward them to all neighbor's CoAs prior MN movement to new subnet in the network.

The overall procedure for the proposed scheme includes two cases, the normal case in which MN-A is only move while the $\mathrm{CN}$ stationary, as follows:

- MN-A expect its movement to new subnet by monitor LGD trigger, then it request the new subnet's CoA using route solicitation message, or by periodic route advertises in overlapping area

- MN-A after getting the CoA, it sends BU $(b=1)$ indication to its $\mathrm{HA}$ to maintains the binding relationship of this MN's home address and neighbor's CoAs 


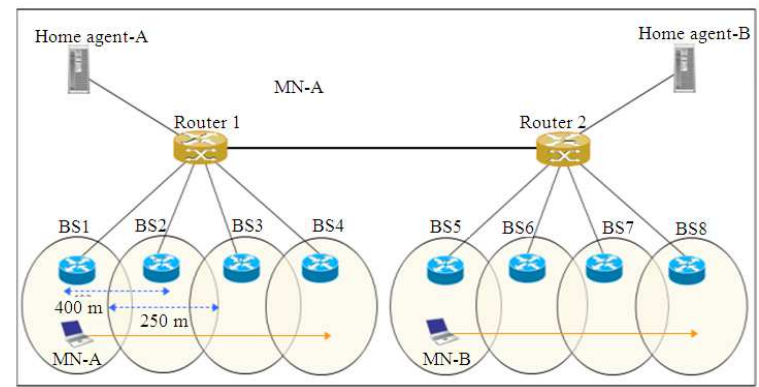

Fig. 4: Network topology experiment

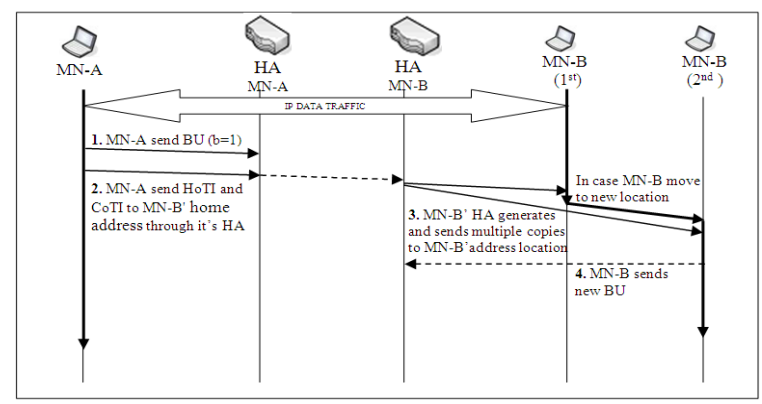

Fig. 5: Signaling flow of the proposed scheme

Table 2: Simulation parameters setting

\begin{tabular}{ll}
\hline Parameters & Values \\
\hline Wireless link bandwidth & $20 \mathrm{Mbps}$ \\
Bandwidth & $80 \mathrm{Mbps}$ \\
L2 Handover & $50 \mathrm{~m} \mathrm{sec}$ \\
UDP packet length & $200 \mathrm{bytes}$ \\
Packet rate & $50 \mathrm{kbps}$ \\
Simulation time & $300 \mathrm{sec}$ \\
\hline
\end{tabular}

- MN-A will sends HoTI message to the stationary $\mathrm{CN}$ through its HA and sends CoTI message directly to $\mathrm{CN}$ in normal routability procedure and complete update location registrations to its HA

The second case, where the $\mathrm{CN}$ is mobile (i.e., MN-B) and moves during MN-A's routability, MN-B's home agent has its neighbor's CoAs. As illustrate in Fig. 5, which described in the following steps:

- $\quad$ MN-A will update its HA by sending a BU $(b=1)$

- MN-A will send both HoTI and CoTI message to the MN-B's home agent through MN-A' HA

- The home agent of the MN-B after receiving these messages, checks its maintained binding relationship. If MN-B has bound MN-B's neighbor's CoAs with its home address in this location registration or delay home registration before MN-A's routability messages arrives. Thus
- The home agent of the MN-B will make multiple copies according to these neighbor's CoAs and send them to MN-B's CoAs locations

In general, the proposed scheme sends a number of copies of control messages to all neighbor's CoAs bound by $\mathrm{MN}$, which cause additional network overhead. Therefore, to overcome this issue, all copies of the control messages which a MN did not roam to their point of attachments will be timeout after a predefined time, in order to reduce network overhead and optimize mobility management cost in MIPv6 domain.

On the other hand, the advantages of the proposed scheme are very understandable when the neighbor's CoAs gotten in the other networks it has been multi bound to the HA. Therefore, one of those control messages copies which sent to this node leastwise reach $\mathrm{MN}$, so inaccessible problem actually resolved, especially, when $\mathrm{MN}$ regularly moves in these networks. Meanwhile, solve successive fast mobility problem. In addition, if the CoA previously received in this network is still valid when a MN moves back to visited network, then $\mathrm{MN}$ can reuse this CoA. This can save the time to get new CoA, which reduces the cost of the correspondent registrations.

Network model: we validate the proposed method and analysis it using ns-2. Our network topology shown in Fig. 4, which include two network each include several Access Routers (ARs); which has two interfaces (i.e., wired interface to the router and wireless interface to $\mathrm{MN}$ ) and each represent different IP subnet.

Simulation model: In this simulation, IEEE $802.11 \mathrm{~b}$ is used as MAC protocol for the ARs, which has a $250 \mathrm{~m}$ transmission range and $80 \mathrm{Mbps}$ link bandwidth and to create overlapping coverage, a $400 \mathrm{~m}$ distance is used between the ARs. Plus, a constant bit rate (CBR) application with $0.05 \mathrm{sec}$ intervals over User Datagram Protocol (UDP). This kind of traffic is usually generated by real-time applications. In the simulation, we have two $\mathrm{MN}$ (i.e., $\mathrm{MN}-\mathrm{A}$ and $\mathrm{MN}-\mathrm{B}$ ) moving linearly at the same time and communicate with each other's starting from the area of first AR towards to next AR's. Two CBR sessions was simulated: from $\mathrm{MN}-\mathrm{A}$ to MN-B and vice versa. The first session start with $20 \mathrm{sec}$ warm up period and the second at $30 \mathrm{sec}$, where the MNs are brought up and the flows begin. The system settings used in the simulations are in Table 2.

Performance metrics: two performance metrics were conducted to evaluate the proposed scheme over the conventional MIPv6, which are the handover latency 
and packet loss rate, respectively. The former is defined in this article as the elapses time between the last packets received by $\mathrm{MN}$ at old access router and the arrival of the first packet at the new access router. Whereas, the later is defined as the ratio of the differences between the total number of packets transmitted and the total number of packets received correctly at the destination to the total number of the packets transmitted at the source. The higher handover latency and packet loss rate causes poorer performance of a network.

\section{RESULTS}

Two different scenarios are conducted in order to investigate the performance of the handover latency and the packet loss rate between the conventional MIPv6 and the proposed schemes, respectively.

Handover latency performance: we investigate the impact of the wireless link delay over the handover latency for both the conventional MIPv6 and the proposed schemes, as shown in Fig. 6. The MN is used with mobility movement velocity $4.17 \mathrm{~m} \mathrm{sec}^{-1}$ and the typical wireless link delay between the MNs and ARs are vary from 10-60 m sec, depending on their distance and channel condition (Lai and Chiu, 2005).

When the wireless link delay between the $\mathrm{MN}$ and its access router increases the difference in handover latency between two schemes also increases. When the wireless link delay is $10 \mathrm{~m} \mathrm{sec}$, the handover latency of the conventional MIPv6 scheme is higher than the proposed scheme and start increasing when wireless link delay increase as shown in Fig. 6. It shows that the proposed scheme has the advantage of degrading performance gracefully in increasing transmission distance as well as the wireless link latency.

Packet loss rate performance: this metric will investigate the impact of the MNs velocity over packet loss rate between the conventional MIPv6 and proposed schemes. As shown in Fig. 7. In the case of successive fast mobility environment when the moving velocity of two nodes MN-A and MN-B reaches $2.78 \mathrm{~m} \mathrm{sec}^{-1}$ (i.e., equivalent to $10 \mathrm{~km} \mathrm{~h}^{-1}$ ) using the conventional MIPv6, the packet loss rate of correspondent registration stay at minimum so the two MNs can communicate with each other normally. When the velocities of the two MNs reach $6.94 \mathrm{~m} \mathrm{sec}^{-1}$ the packet loss rate of the correspondent registration begins increasing.

However, when the MN velocity reaches to $9.72 \mathrm{~m} \mathrm{sec}^{-1}$, it will highest failure rate of correspondent registration between MN-A and MN-B which cause high packet loss in the conventional MIPv6.

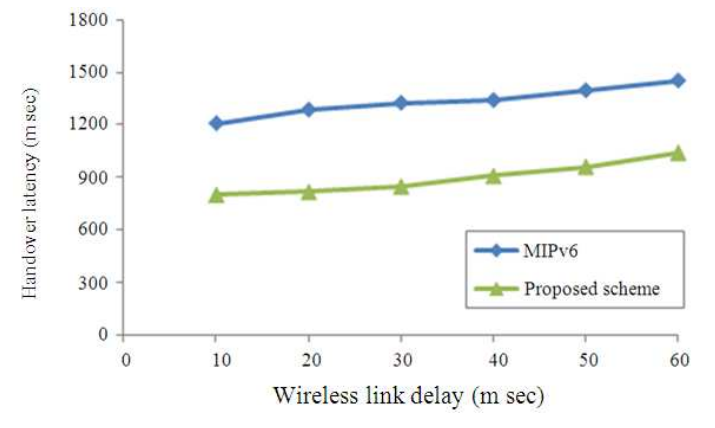

Fig. 6: Comparison of the handover latency between the conventional MIPv6 and proposed schemes

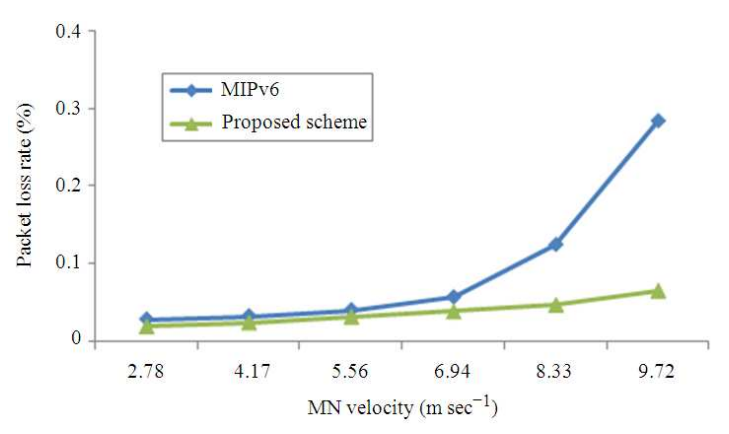

Fig. 7: Comparison of the packet loss rate between the conventional MIPv6 and proposed schemes

In addition, using proposed scheme in HA, when the moving velocity for both $\mathrm{MN}-\mathrm{A}$ and $\mathrm{MN}-\mathrm{B}$ reaches $9.72 \mathrm{~m} \mathrm{sec}^{-1}$, failure rate of correspondent registration is less than the conventional MIPv6 and the two MNs can communicate normally with tolerable packet loss.

\section{DISCUSSION}

The discussion on this scheme has been investigated into the aspect that could give an impact onto their performance. In this scheme the HA forward the control packet to all the available CoA and before the completion of the checking the uniqueness of the address using $\mathrm{DAD}$, the $\mathrm{MN}$ can receive all data packet, so the total handover latency caused by MN registration is reduced. The advantages of the proposed scheme are obvious when the all neighbor's CoAs has been multi bound to the HA. Therefore, one of the controls messages copies which sent to this node leastwise reach $\mathrm{MN}$, especially, when MN continually moves in these networks. Another advantage, to save the time to get a new $\mathrm{CoA}$; if the $\mathrm{CoA}$ previously received in this network is still valid when a MN moves back to the revisit network, then $\mathrm{MN}$ can reuse this CoA, which reduces the cost of the correspondent registrations. 
The results also show a tolerable packet loss over the conventional MIPv6. This scheme can satisfy to some extent the requirement of the delay sensitive services better than the conventional MIPv6 scheme.

\section{CONCLUSION}

In this article, a scheme for resolving the simultaneous mobility problems and reducing the handover latency is proposed. In addition, the proposed scheme can effectively solved successive fast mobility problem and reduces the cost of correspondent registrations initiated by other MNs. Simulation result shows lower handover latency and tolerable packet loss for the proposed scheme over the conventional MIPv6 protocol. It can be greatly used in the areas which communication localized with a fixed area. As future study, our future plan to evaluate and improve the proposed scheme by making additional simulation investigations to overcome other issues such as packets forwarding cost, network load and supporting QoS to satisfy the requirements for the real-time sensitive applications.

\section{REFERENCES}

Badis, H. and K.A. Agha, 2003. An efficient mobility management in wireless overlay network. Proceeding of the 14th IEEE Personal Indoor and Mobile Radio Communication, Sept. 7-10, IEEE Xplore Press, USA., pp: 2500-2504. DOI: 10.1109/PIMRC.2003.1259173

Daley, G., B. Pentland and R. Nelson, 2003. Effects of fast router advertisement on mobile IPv6 handovers. Proceeding of the 8th IEEE International Symposium on Computers and Communication, June 30-July 3, IEEE Xplore Press, USA., pp: 557-562. DOI: 10.1109/ISCC.2003.1214177

IETF-mobileip, 2004. IETF Mobile IP Working Group (mobileip). IETF. http://datatracker.ietf.org/wg/mip6/charter/

Johnson, D., C. Perkins and J. Arkko, 2004. RFC3775mobility support in IPv6. Network Working Group. http://www.faqs.org/rfcs/rfc3775.html

Klein, T.E. and S.J. Han, 2004. Assignment strategies for mobile data users in hierarchical overlay networks: Performance of optimal and adaptive strategies. IEEE J. Select. Areas Commun., 22: 849-861. DOI: 10.1109/JSAC.2004.826922

Lai, W.K. and J.C. Chiu, 2005. Improving handoff performance in wireless overlay networks by switching between two-layer IPv6 and one-layer IPv6 addressing. IEEE J. Select. Areas Commun., 23: 2129-2133. DOI: 10.1109/JSAC. 2005.856828
Liu, Q., S. Li, H. He and B. Wang, 2006. A multibinding solution for simultaneous mobility of MIPv6. Proceeding of the IEEE International Workshop on Service-Oriented System Engineering, Oct. 2006, IEEE Xplore Press, Shanghai, pp: 143-146. DOI: 10.1109/SOSE.2006.1

Ma, W. and Y. Fang, 2004. Dynamic hierarchical mobility management strategy for mobile IP networks. IEEE J. Select. Areas Commun., 22: 664-676. DOI: 10.1109/JSAC.2004.825968

Malki, K.E., P.R. Calhoun, T. Hiller, J. Kempf and P.J. McCann, 2002. Low latency handoffs in mobile IPv4. Mobile IP Working Group. http://tools.ietf.org/html/draft-ietf-mobileiplowlatency-handoffs-v4-01

Narten, T., E. Nordmark and W. Simpson, 1998. Neighbor discovery for IP version 6 (IPv6). IETF.

Salamah, M., F. Tansu and N. Khalil, 2003. Buffering requirements for lossless vertical handoffs in wireless overlay networks. Proceeding of the 57th IEEE Semiannual Vehicular Technology Conference, Apr. 22-25, IEEE Xplore Press, USA., pp: 1984-1987. DOI: 10.1109/VETECS.2003.1207172

Soliman, H., C. Castelluccia, K. El Malki and L. Bellier, 2005. Hierarchical MIPv6 Mobility management (HMIPv6). Network Working Group. http://www.rfc-archive.org/getrfc.php?rfc=4140

Wong, K.D. and A. Duttay, 2005. Simultaneous mobility in MIPv6. Proceeding of the IEEE Electro Information Technology Conference, May 22-25, IEEE Xplore Press, Lincoln, NE., pp: 1-5. DOI: 10.1109/EIT.2005.1626976

Wong, K.D., A. Dutta, K. Young and H. Schulzrinne, 2003a. On handling simultaneous mobility. IEEE Milcom.

Wong, K.D., A. Dutta, K. Young and H. Schulzrinne, 2003b. Managing simultaneous mobility of IP hosts. Proceeding of the IEEE Military Communications Conference, Oct. 13-16, IEEE Xplore Press, USA., pp: 785-790. DOI: 10.1109/MILCOM.2003.1290212

Wong, K.D., A. Duttay, H. Schulzrinnez and K. Young, 2007. Simultaneous mobility: Analytical framework, theorems and solution. Wireless Commun. Mobile Comput., 7: 623-642. DOI: 10.1002/wcm.389 